\title{
Research on Equipment of Concrete Mixing Plant Based on The Internet of Things Technology
}

\author{
Hualong Cai ${ }^{1}$, Ming Ouyang ${ }^{1, *}$, Chengyu $\mathrm{Zhu}^{1}$ and Zeming Xiao ${ }^{1}$ \\ ${ }^{1}$ School of Civil Engineering, Jiaying University, Meizhou, Guangdong, 514015, China
}

\begin{abstract}
Concrete plays an important role in modern engineering construction. Concrete quality relates directly to the quality of the project life cycle and the safety of people's lives and property. Based on the theories and methods of IOT technology, this article applies IOT technology to concrete mixing plant equipment, creatively proposes an integrated system of concrete mixing plants, to control the whole process and the whole cycle of concrete production, and improve the quality of concrete. The practical application of the system has greatly improved concrete production efficiency and production quality.
\end{abstract}

\section{Background of research}

With the gradual maturity of IOT technology, the Internet of Things technology is becoming more and more widely used in the construction machinery industry. As an important building material in modern engineering construction, the quality of concrete is directly related to the quality of the project life cycle and the safety of people's lives and property. However, concrete mixing station apparatus as a main device to produce concrete, but faced with the artificial inaccurate metering data, production process control cycle is not in place and so on [1].

Based on this situation, this article proposes to integrate the Internet of Things technology with concrete mixing plant equipment, develop a concrete mixing plant IOT integrated system, realize the full process and full cycle control of the concrete mixing plant equipment, and ensure the production of high-quality concrete.

\section{System composition and functions}

The concrete mixing plant system consists of six subsystems: batching system, mixing system, intelligent control system, gas control system, dust removal system and material control system [2]. It has been shown in figure 1 .

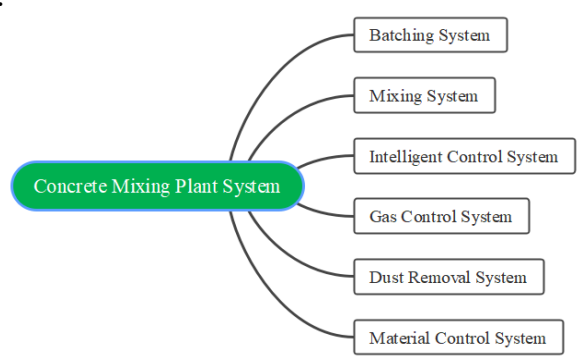

Figure 1. System Composition

\subsection{Batching Systems}

Staffs input the corresponding batching ratio in the system page, and the connected controller will accept the induction and then make the material enter into the measuring scale by vibrating the liner and adjusting the decompression plate, while the sensor on the scale will display the weight of the material on the scale in the system to realize the function of automatic over-weighing and deducting the scale, and lack of weighing and replenishing the scale to ensure the accuracy of material measurement.

\subsection{Mixing System}

Staffs enter the mixing time on the system page to remotely control the controller on the mixer, thus controlling the mixer for automatic mixing and ensuring precise control of the mixing time to avoid the concrete mixing time being too long or too short and affecting the quality of the concrete.

\subsection{Intelligent Control System}

The intelligent control system can control each sub-system of the concrete mixing system wirelessly and remotely, with three working modes: fully automatic, semiautomatic and manual. At the same time, it can check the operation status of the equipment in real time through sensors in all aspects and upload the data such as batching ratio and mixing time to the database for big data analysis, which helps to improve the quality of concrete production.

\footnotetext{
$\overline{{ }^{*} \text { Corresponding author's email: whucad }} @$ whu.edu.cn
} 


\subsection{Gas Control System}

During the batching process of the mixing plant, the zeroing output is carried out through the pneumatic control system, which automatically corrects the falling difference, effectively controls the accuracy of the measurement, can accurately adjust and modify the setting value of each material, and is equipped with equipment fault detection and light alarm device.

\subsection{Dust Removal System}

This system adopts pulse back-blowing dust removal technology to fully automate the dust removal of the equipment by controlling the timing of the pulse controller.

\subsection{Material Control System}

The materials are stored through steel structure containers, and the temperature and humidity inside the containers are monitored through the sensors inside the containers, and the temperature and humidity of the containers are controlled by this system to ensure the quality of the materials. This system automatically feeds powder and aggregate through belt machine, which controls the amount of dust generated; water and liquid admixture are automatically transported through water pump. The weighing of all materials is controlled by electronic scale and microcomputer to ensure the weighing accuracy of aggregate, cement, powder, water and admixture.

\section{Further innovative use of IOT technology in concrete mixing plant equipment}

Based on the concrete mixing plant system, this paper innovatively proposes an integrated IOT system for concrete mixing plant by combining IOT technology in a deeper level, which contains five new systems: concrete mixing reservation system, data collection and analysis management system, remote monitoring system, equipment maintenance system and office OA system. It has been shown in figure 2 .

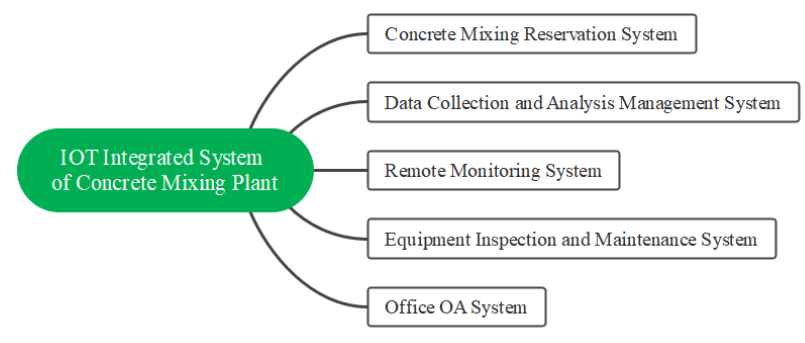

Figure 2. IOT integrated system of concrete mixing plant

\subsection{Concrete mixing reservation system}

According to the actual construction progress, the construction personnel can access the concrete mixing reservation system through various ways such as concrete mixing plant integrated IOT system machine, mobile app or website, and independently choose the specified time period to make reservation for concrete mixing plant equipment.

\subsection{Data collection and analysis management system}

When the concrete mixing plant is in operation, the hardware devices such as sensors and monitors are automatically turned on simultaneously to monitor and collect data such as mortar volume, water consumption and air pressure, and upload them to the system's background database. After that, the system automatically stores, backs up and analyzes the data, and reflects the operation of the concrete mixing plant to the user in real time in the form of bar graphs, curve graphs and other dynamic methods such as sound, light and color.

\subsection{Remote monitoring system}

The system is composed of two parts: monitoring information platform and monitoring equipment terminal. Among them, the equipment monitoring terminal is mainly composed of monitoring cameras, GPRS remote wireless communication system and other equipment. The monitoring data is uploaded to the information platform in real time using the sensors installed on the equipment, and the administrator monitors the operating status of the equipment in real time through the surveillance cameras and the monitoring information platform at the same time [3]. The monitoring information platform can automatically store, backup and analyze the data uploaded at the construction site, and give early warning and alarm alerts when the equipment is in an abnormal working state [4].

\subsection{Equipment inspection and maintenance system}

If the operator finds that the equipment is damaged during the process of concrete production, he can enter the equipment maintenance system to report the equipment repair, which will automatically convey the equipment repair information to the maintenance personnel and remind the maintenance personnel to repair the equipment as soon as possible. After the equipment maintenance is completed, the maintenance personnel will register the information of equipment maintenance completion in this system, and after that, the system will automatically release the information of equipment maintenance completion and remind the construction personnel.

\subsection{Office OA system}

This system releases information on equipment operation, equipment reservation and equipment maintenance on important sections, scrolling screens and business information tabs on the information platform, making it easy for relevant staff to understand the operation of equipment. 


\section{System architecture}

\subsection{Software structure}

The system software is horizontally divided into a fourlayer structure: resource layer, core business layer,

platform layer [5] and data access layer, and vertically a security management system layer. Resource layer including surveillance cameras, sensors, alarms and so on. Core business layer including batching system, mixing system, intelligent control system and so on. The platform layer includes unified service interface, data sharing, and system operation and maintenance. It has been shown in figure 3 .

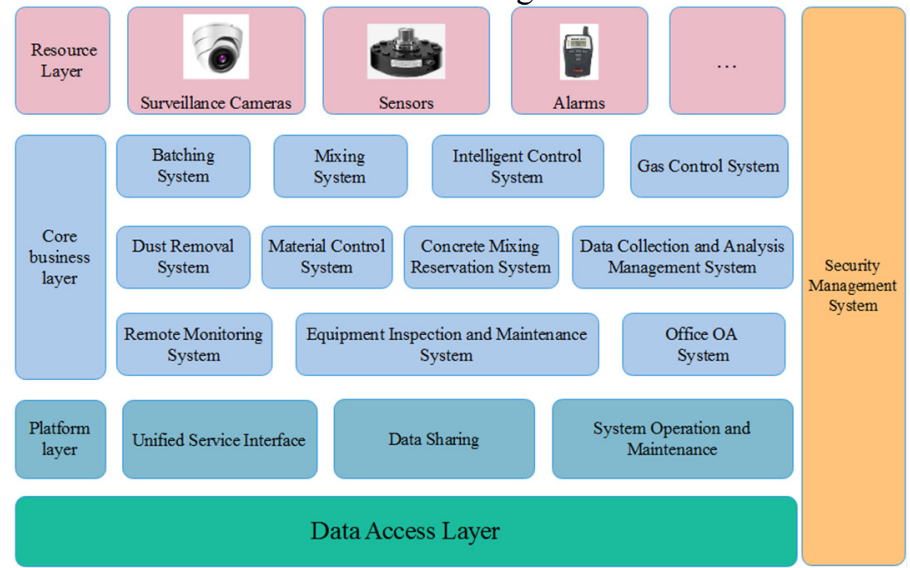

Figure 3. Software Structure

\subsection{Hardware structure}

on cloud service, which stores the data of production site, supervisory unit, mixing station test room and owner's server data center in the cloud server for easy retrieval by the user side. It has been shown in figure 4.

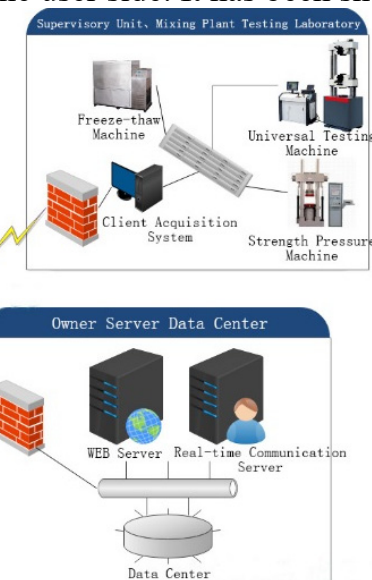

Figure 4. Network Topology Diagram

\section{System implementation}

\subsection{System main interface}

The backend administrator uploads the data of the user (not the system administrator) and notifies the user of the initial password and other information, but the individual cannot register the account alone. The login interface has been shown in figure 5 .

\begin{tabular}{|c|c|}
\hline \multicolumn{1}{|c|}{ IOT Integrated System of Concrete } \\
Mixing Plant
\end{tabular}

Figure 5. Login Interface

After the user logs in, the user will see the system interface divided into four areas. The top is the title bar, the left is the function navigation area, the right is the content display area, and the bottom is the copyright declaration area. It has been shown in figure 6 . 


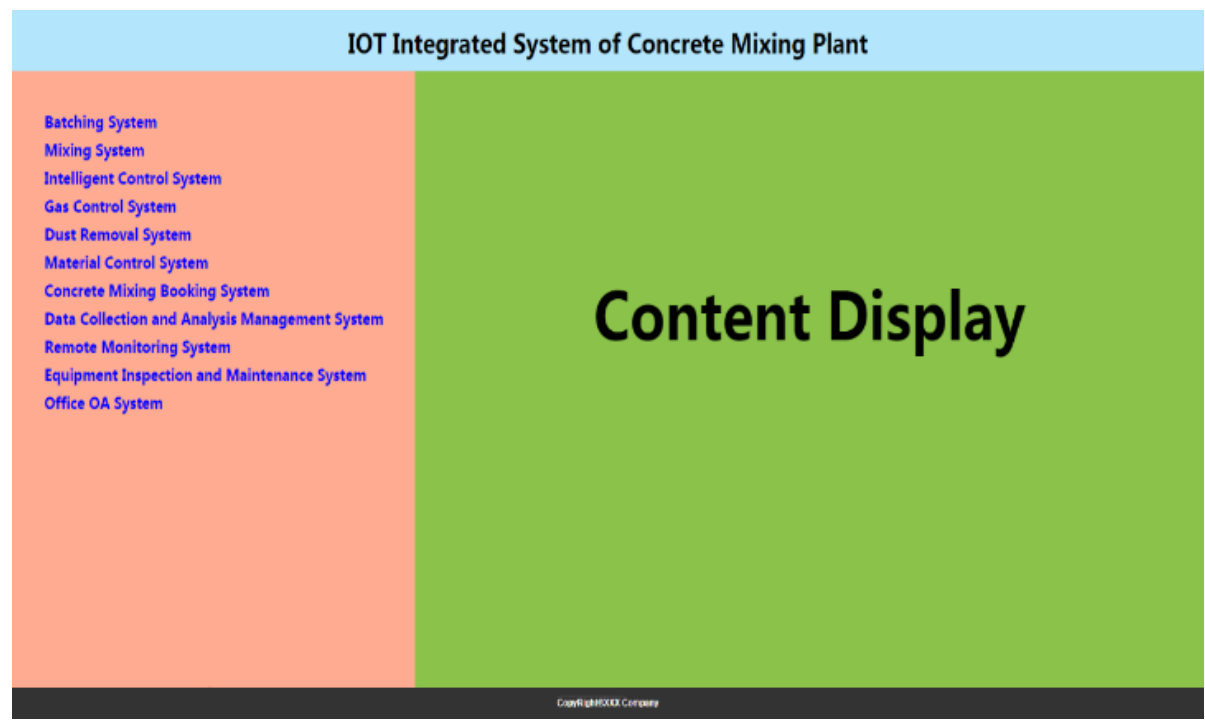

Figure 6. User Interface Partition

\subsection{Related system interface}

Uploading photos related to the operation of the concrete mixing plant equipment based on the PHP development environment, part of the code of which is shown below:

$<$ script type="text/javascript" $>$

window.onload $=$ function () \{

var fileTag $=$

document.getElementById('file');

fileTag.onchange $=$ function ()\{

var file $=$ fileTag.files $[0]$

if(!/image $\backslash \mathrm{w}+/$.test(file.type $)$ )

alert("Hello, you choose not the picture,

here should choose to use the picture "); return false;

var fileReader $=$ new FileReader(); fileReader.onloadend $=$ function () \{

fileReader.DONE) \{ if $($ fileReader.readyState $==$

document.getElementById('img').setAttribute('src', fileReader.result); \} \};

fileReader.readAsDataURL(file); $\} ;\} ;</$ script $>$

\section{Conclusions and prospects}

This paper applies IOT technology to concrete mixing plant equipment and creatively proposes a concrete mixing plant IOT integrated system, which can control the whole process and cycle of concrete production through information transmission between platforms such as data center and production site, and then improve the quality of concrete, which is conducive to improving the quality of engineering and promoting the high-speed development of China's infrastructure construction. Further promoting the application of IOT technology in production and construction equipment is an inevitable trend to fully realize the modernization of engineering in the future. This paper suggests to continue to strengthen the application and research of IOT technology in construction equipment in the future, and give full play to the unique advantages of IOT technology in construction equipment.

\section{References}

1. Zheng, K.R. (2014) An analysis of factors affecting the quality of commercial concrete production. Construction Supervision Inspection and Costing., 07:61-64.

2. Jiang, D., Liao, C., Zhang, Z.Q., Mo J.F. (2021) Research on real-time diagnosis of concrete mixing plant metering sensor faul. Construction Machinery Technology and Management., 34:109-114.

3. Shao, D., Zhang, F., Du C.C. (2017) Application of Internet of things technology on pipeline construction equipment management. China Chemical Trade., 09:117.

4. Wang, B. (2021) Research on construction hoist safety supervision platform based on Internet of Things technology. Construction Machinery., 52:1722.

5. Li, X., Li, N., Zhang, Y.N., Li, S.Q. (2021) Application of GIS and Internet of Things technology in the construction of smart sites. Mapping and spatial geographic information., 44:159-161. 